OPEN ACCESS

Edited by:

Young Kwok,

University of Maryland Medical Center,

United States

Reviewed by:

Shahed Nicolas Badiyan,

Washington University in St. Louis,

United States

John E. Mignano,

Tufts University School of Medicine,

United States

*Correspondence:

sung Ho Moon

shmoon@ncc.re.kr

Specialty section:

This article was submitted to

Radiation Oncology

a section of the journal

Frontiers in Oncology

Received: 23 April 2021

Accepted: 20 May 2021

Published: 21 June 2021

Citation:

Suh Y-G, Bayasgalan U, Kim HT,

Lee JM, Kim MS, Lee Y, Lee DY, Lee SU, Kim TH and Moon SH (2021) Photon Versus Proton Beam Therapy for T1-3 Squamous Cell Carcinoma of

the Thoracic Esophagus Without

Lymph Node Metastasis.

Front. Oncol. 11:699172.

doi: 10.3389/fonc.2021.699172

\section{Photon Versus Proton Beam Therapy for T1-3 Squamous Cell Carcinoma of the Thoracic Esophagus Without Lymph Node Metastasis}

\author{
Yang-Gun Suh ${ }^{1}$, Unurjargal Bayasgalan ${ }^{2}$, Heung Tae $\mathrm{Kim}^{3}$, Jong Mog Lee ${ }^{4}$, \\ Moon Soo Kim ${ }^{4}$, Youngjoo Lee ${ }^{3}$, Doo Yeul Lee ${ }^{1}$, Sung Uk Lee ${ }^{1}$, Tae Hyun Kim ${ }^{1}$ \\ and Sung Ho Moon ${ }^{1 *}$ \\ 1 Proton Therapy Center, Research Institute and Hospital, National Cancer Center, Goyang, South Korea, 2 Department of \\ Radiation Oncology, National Cancer Center, Ulaanbaatar, Mongolia, ${ }^{3}$ Department of Internal Medicine, Research Institute \\ and Hospital, National Cancer Center, Goyang, South Korea, ${ }^{4}$ Department of Thoracic Surgery, Research Institute and \\ Hospital, National Cancer Center, Goyang, South Korea
}

Background and Purpose: We compared treatment outcomes and toxicities of photon radiotherapy versus proton beam therapy (PBT) and evaluated radiation field effects for $\mathrm{T} 1-$ 3 squamous cell carcinoma of the thoracic esophagus (EC) without lymph node metastasis.

Methods: Medical records of 77 patients with T1-3NOMO thoracic EC treated with radiotherapy between 2011 and 2019 were retrospectively analyzed. Among these patients, 61 (79.2\%) individuals had T1 EC. The initial clinical target volume encompassed the whole esophagus with or without supraclavicular and/or abdominal lymph nodes (extended-field radiotherapy; 67 patients, $87.0 \%$ ) or the area $3-5 \mathrm{~cm}$ craniocaudally and $1-2 \mathrm{~cm}$ radially from the gross tumor volume (involved-field radiotherapy; 10 patients, $13.0 \%$ ). The final clinical target volume included margins of at least $1 \mathrm{~cm}$ from the gross tumor volume, with total radiation doses of 50-66 (median, 66) cobalt gray equivalent. Three-dimensional conformal radiotherapy, intensity-modulated radiotherapy, and PBT were used in twenty-four, five, and forty-eight patients, respectively. Concurrent chemotherapy was administered to 17 (22.0\%) patients overall and only five (8.0\%) T1 patients.

Results: PBT showed significantly lower lung and heart radiation exposure in mean dose, V5, V10, V20, and V30 than photon radiotherapy. The median follow-up for all patients was 46 (interquartile range, 22-72) months. The 5-year progression-free survival and overall survival rates were 56.5 and $64.9 \%$, respectively, with no significant survival difference between photon radiotherapy and PBT. In patients with T1 EC, 5-year progression-free survival and overall survival rates were 62.6 and $73.5 \%$, respectively. 
Conclusions: Extended-field radiotherapy using modern radiotherapy techniques without chemotherapy showed satisfactory clinical outcomes for lymph node-negative T1 EC.

Keywords: esophageal cancer, proton beam therapy, 3-dimensional radiotherapy, intensity-modulated radiotherapy, endoscopic submucosal dissection, lymph node metastasis, organs at risk

\section{INTRODUCTION}

The esophagus has abundant and complex lymphatic channels that begin from the submucosal layer and the outer layers differently communicating intramurally and extramurally (1). Therefore, lymph node (LN) metastasis frequently develops after treatment of esophageal cancer (EC), even in clinically LNnegative T1 superficial EC. Nodal skip metastasis, which is found far from the original tumor location, is a common pattern of disease progression in EC. The LN metastasis rates for pathologic T1, T2, and T3 EC after surgery are 15-20, 40-50, and $70-75 \%$, respectively $(2,3)$. Accordingly, surgery for thoracic $\mathrm{EC}$ frequently requires a meticulous $\mathrm{LN}$ dissection encompassing the cervico-thoracic-abdominal area in addition to subtotal or total esophagectomy. This extensive surgical procedure for EC can result in a postoperative mortality rate of $0-7 \%$ (4-6), and complications include laryngeal nerve palsy and gastrointestinal dysfunction $(7,8)$.

Radiotherapy (RT) with or without concurrent chemotherapy is often accepted for definitive treatment of T1 EC. The use of involved-field RT (IFRT) versus elective nodal irradiation (ENI) is controversially discussed, as skipped LN metastasis substantially occurs outside of the RT field boundary after IFRT, even with chemotherapy. Moreover, these regional recurrences are difficult to salvage, whereas most cases of local recurrence in the esophagus or metachronous primary EC are successfully salvaged with endoscopic resection or surgery (911). Therefore, the necessity of extended-field RT (EFRT) has been increasingly emphasized (12). We also previously reported a study of 24 patients with superficial EC presenting satisfactory outcomes after EFRT without chemotherapy targeting at least the entire esophagus (13).

Modern photon RT techniques including three-dimensional conformal RT (3D-CRT) and intensity-modulated RT (IMRT) have been widely used for EC treatment, but there are risks of acute or delayed cardiopulmonary toxicity due to substantial radiation exposure to the lung and the heart. Proton beam therapy (PBT) has a unique physical characteristic known as the Bragg peak, which allows the rapid elimination of a radiation dose after passing through the tumor target. PBT has been expected to reduce radiation-related toxicity effects by decreasing unintended exposure of healthy organs to radiation. Previous dosimetric studies have shown that in comparison to IMRT, PBT significantly decreases the radiation dose to the lung and heart $(14,15)$. Moreover, a recent study demonstrated that PBT significantly reduces the frequency of severe lymphopenia, which is known as a poor prognostic factor, compared to photon RT in neoadjuvant chemoradiation for EC (16).
In the present study, we compared treatment outcomes and toxicities of photon RT (3D-CRT and IMRT) with those of PBT and evaluated RT field issue in patients with T1-T3 thoracic EC without LN metastasis, the majority of whom underwent EFRT without chemotherapy.

\section{MATERIALS AND METHODS}

\section{Patients}

The medical records of 77 patients with cT1-3N0M0 squamous cell carcinoma of thoracic esophagus who underwent definitive RT with or without chemotherapy at the National Cancer Center in Republic of Korea between November 2011 and November 2019 were retrospectively reviewed. Baseline characteristics included the medical history and physical examination results. Laboratory measurements included complete blood cell count and blood biochemistry measurements. For staging, esophagogastroduodenoscopy with Lugol staining, chest computed tomography (CT), endoscopic ultrasonography, and ${ }^{18}$ F-fluorodeoxyglucose positron emission tomography (FDGPET) were performed. The clinical stage was based on the $7^{\text {th }}$ edition of the American Joint Committee on Cancer (AJCC) TNM classification. This study was approved by the Institutional Review Board of National Cancer Center in Republic of Korea (NCC2020-0043).

\section{Endoscopic Resection of T1 EC After Risk Assessment}

Our multidisciplinary esophageal cancer oncology clinic by radiology, gastroenterology, radiation oncology, thoracic surgical oncology, medical oncology, and radiation oncology subspecialists recommends that some patients with T1 EC who underwent endoscopic submucosal dissection (ESD) in our hospital require further treatment with esophagectomy or RT with or without chemotherapy. Indications for further treatment are incompleteness of ESD including invasion of the submucosa or lymphovascular space by the tumor, or close or positive resection margins. Patients who were medically inoperable or those who were reluctant to undergo surgery after ESD for T1 EC were treated with RT, as were those treated without ESD for T1 EC.

\section{Radiotherapy}

RT was delivered using megavoltage photon beams or proton beams. A conventional fractionation schedule with a fraction size of two cobalt gray equivalent (CGE) was used for all patients. The gross tumor volume (GTV) was defined as the tumor volume 
visualized on chest CT or FDG-PET or as the area marked with clips located near the proximal and distal ends of the primary tumor or ESD bed by a specialized upper gastrointestinal endoscopist before undergoing an RT simulation CT. In patients with esophageal clips, clip migration or removal was verified before the start of the RT.

The shrinking field technique was used for all patients. For IFRT, the initial clinical target volume (CTV2) extended $5 \mathrm{~cm}$ craniocaudally and $1-2 \mathrm{~cm}$ radially from the GTV. For EFRT, the CTV2 encompassed the entire esophagus and regional LN group including the pretracheal, retrotracheal, paratracheal, subcarinal, and peri-esophageal LNs with or without the supraclavicular LN (SCN) and a part of the abdomen including the paracardial, left gastric, and celiac LNs. The final clinical target volume (CTV1) covered the GTV with a margin of at least $1-2 \mathrm{~cm}$ craniocaudally and radially. Subsequently, the planning target volume (PTV) was defined as the CTV plus $0.5-1 \mathrm{~cm}$. Total doses of 38-46 CGE (median 44 CGE) and 50-66 CGE (median 66 CGE) were delivered once daily to the initial PTV (PTV2) and the final PTV (PTV1), respectively.

The 3D-CRT and PBT plans were generated using the Eclipse $^{\mathrm{R}}$ planning system (Varian Medical System, Palo Alto, $\mathrm{CA})$. During the 3D-CRT and PBT planning process, anteriorposterior parallel-opposed fields were usually selected in the initial phase to reduce the dose to the lungs, followed by anterior-posterior oblique fields or two posterior oblique fields in the boost phase to limit the dose to the spinal cord. For PBT, passive-scattering, uniform scanning, or pencil beam scanning techniques were used. The IMRT plan was generated using the Eclipse $^{\mathrm{R}}$ planning system or the Tomotherapy ${ }^{\mathrm{R}}$ Planning System (Accuray, Sunnyvale, CA), and 3-5 fields were used with a dynamic multileaf collimator or helical tomotherapy unit. Photon beams of 6-15 MV and proton beams of $230 \mathrm{MeV}$ were used.

\section{Chemotherapy}

Capecitabine with or without platinum was the most commonly used regimen $(\mathrm{n}=11)$, followed by bolus 5 -fluorouracil (5-FU, $\mathrm{n}=3$ ) and paclitaxel-based chemotherapy $(\mathrm{n}=3)$. Two cycles of 5 -FU- or a capecitabine-based regimen with or without cisplatin were delivered starting on days 1 and 22. Capecitabine was administered orally twice daily for 14 days at a dose of 2,500 $\mathrm{mg} / \mathrm{m} 2 /$ day. 5 -FU was administered at $600-700 \mathrm{mg} / \mathrm{m} 2 /$ day on days 1-4 and days 22-25, and cisplatin was infused intravenously at $60-75 \mathrm{mg} / \mathrm{m} 2$ on days 1 and 22 . Paclitaxel $(50 \mathrm{mg} / \mathrm{m} 2)$ and carboplatin [area under the curve $(\mathrm{AUC})=2$ ] were administered weekly for 5-6 cycles during RT. No patient underwent neoadjuvant chemotherapy.

\section{Follow-Up and Statistical Analysis}

Patients were examined weekly to evaluate acute treatmentrelated toxicities and the patients' general condition during RT; they were then followed up with esophagogastroduodenoscopy or chest CT every 3 months for the first 2 years and every 6 months thereafter. Acute and chronic treatment-related toxicities were graded based on the Common Terminology Criteria for Adverse Events (CTCAE), version 4.0. Treatment responses were evaluated according to the Revised Response Evaluation Criteria in Solid Tumors (RECIST) Guidelines, version 1.1.

For the patterns of failure analysis, recurrences were subdivided into (1) local recurrence, progression of the primary tumor or occurrence of metachronous primary EC; (2) regional recurrence, spread to regional LNs based on the 7 th edition of the AJCC TNM classification; and (3) distant LN or solid organ metastasis such as to the liver or lungs. Recurrences were also classified depending on their location as within (infield), at the boundary of (marginal), or outside (outfield) the initial RT field.

Survival duration was calculated from the first day of RT until the day of the last follow-up or the occurrence of events such as local failure, regional failure, distant failure, or death. The chisquare test or Fisher's exact test was used to assess the measures of association between the risk factors and treatment-related toxicities as appropriate. The Wilcoxon rank-sum test was used to compare differences in continuous variables between two groups. Survival rates were calculated with the Kaplan-Meier method, and survival differences between groups were analyzed using the log-rank test. Statistical analyses were conducted using R Statistical Software version 4.0.3 (R Foundation for Statistical Computing, Vienna, Austria). The "gganatogram" package was used to visualize the sites of recurrences (17).

\section{RESULTS}

\section{Patient and Treatment Characteristics}

The median age of all patients was 72 years (range, $44-89$ years), with $70(90.9 \%)$ men and seven $(9.1 \%)$ women. Most patients (92.2\%) had an Eastern Cooperative Oncology Group (ECOG) performance status score of $0-1$. The tumors were in the upper, mid-, and lower thoracic esophagus in seven (9.1\%), 34 (44.2\%), and $36(46.7 \%)$ patients, respectively. Most patients $(n=61$, 79.2\%) presented with T1 tumors, and eight (10.4\%) and eight (10.4\%) patients presented with T2 and T3 tumors, respectively. The median tumor size was $2.5 \mathrm{~cm}$ and ranged from 0.6 to $17 \mathrm{~cm}$. Photon RT and PBT were administered to 29 (37.7\%) and 48 (62.3\%) patients, respectively. The baseline patient and tumor characteristics are summarized in Table $\mathbf{1}$.

The SCN and a part of the abdomen were irradiated in 53 (68.8\%) and $44(57.1 \%)$ patients, respectively. Abdominal LN irradiation was more frequently performed in the PBT group (72.9 versus $31.0 \%, p=0.001$ ). Twenty-six patients received RT after ESD due to submucosal invasion and close or involved resection with margins less than $1 \mathrm{~mm}$. Concurrent chemotherapy was administered to five $(8.2 \%)$ patients with $\mathrm{T} 1$ tumors and 12 (75.0\%) patients with T2-3 tumors. Details about the treatments are summarized in Table 2 . The percentage of patients who received chemotherapy did not differ between groups.

\section{Dosimetric Analysis}

Radiation exposures to the lung and heart are compared between groups in Figure 1. Compared to photon RT, PBT significantly 
TABLE 1 | Patient characteristics.

\begin{tabular}{|c|c|c|c|c|}
\hline Characteristics & All, No. (\%) & Photon RT, No. (\%) & PBT, No. (\%) & $p$ value \\
\hline No. of patients & 77 (100) & $29(37.7)$ & $48(62.3)$ & \\
\hline \multicolumn{5}{|l|}{ Age (years) } \\
\hline Median & $72(44-89)$ & 73 (44-89) & $69(47-85)$ & 0.203 \\
\hline$\leq 69$ & $33(42.9)$ & $9(31.0)$ & $24(50.0)$ & 0.164 \\
\hline$\geq 70$ & $44(57.1)$ & $20(69.0)$ & $24(50.0)$ & \\
\hline \multicolumn{5}{|l|}{ Sex } \\
\hline Male & 70 (90.9) & $26(89.7)$ & $19(91.7)$ & 1.000 \\
\hline Female & $7(9.1)$ & $3(10.3)$ & 4 (8.3) & \\
\hline \multicolumn{5}{|l|}{ Performance status } \\
\hline ECOG 0 & $33(42.9)$ & 9 (31.0\%) & 24 (50.0\%) & 0.263 \\
\hline ECOG 1 & 38 (49.3) & 17 (58.6\%) & 21 (43.8\%) & \\
\hline ECOG 2 & $6(7.8)$ & $3(10.3 \%)$ & $3(6.2 \%)$ & \\
\hline \multicolumn{5}{|l|}{ Histologic grade } \\
\hline WD & $7(9.1)$ & 3 (10.3\%) & $4(8.3 \%)$ & 0.168 \\
\hline MD & $41(53.2)$ & 17 (58.6\%) & 34 (70.8\%) & \\
\hline PD & $16(20.8)$ & $6(20.7 \%)$ & $10(20.8 \%)$ & \\
\hline Unknown & 3 (3.9) & $3(10.3 \%)$ & $0(0.0 \%)$ & \\
\hline \multicolumn{5}{|l|}{ Tumor location } \\
\hline Upper thoracic & $7(9.1)$ & 3 (10.3\%) & $4(8.3 \%)$ & 0.023 \\
\hline Middle thoracic & $34(44.2)$ & $18(62.1 \%)$ & $16(33.3 \%)$ & \\
\hline Lower thoracic & $36(46.7)$ & $8(27.6 \%)$ & 28 (58.3\%) & \\
\hline \multicolumn{5}{|l|}{ T stage } \\
\hline $\mathrm{T} 1$ & $61(79.2)$ & 21 (72.4\%) & 40 (83.3\%) & 0.713 \\
\hline $\mathrm{T} 2$ & $8(10.4)$ & 3 (10.3\%) & 5 (10.4\%) & \\
\hline T3 & 8 (10.4) & 5 (17.2\%) & $3(6.2 \%)$ & \\
\hline \multicolumn{5}{|l|}{ Tumor size $(\mathrm{cm})$} \\
\hline Median & $2.5(0.6-17)$ & $2.5(0.6-8)$ & $2.75(1.0-17.0)$ & 0.869 \\
\hline$<3$ & 40 (51.9) & $16(55.2 \%)$ & 24 (50.0\%) & 0.838 \\
\hline$\geq 3$ & $37(48.1)$ & 13 (44.8\%) & 24 (50.0\%) & \\
\hline
\end{tabular}

$R T$, radiotherapy; PBT, proton beam therapy; ECOG, Eastern Cooperative Oncology Group; SCC, squamous cell carcinoma; WD, well differentiated; MD, moderately differentiated; PD, poorly differentiated.

TABLE 2 | Treatment characteristics.

\begin{tabular}{|c|c|c|c|c|}
\hline Characteristics & All, No. (\%) & Photon RT, No. (\%) & PBT, No. (\%) & $p$ value \\
\hline No. of patients & $77(100)$ & $29(37.7)$ & $48(62.3)$ & \\
\hline \multicolumn{5}{|l|}{ Radiation dose } \\
\hline Median, CGE & $66(50-66)$ & $64(56-66)$ & $66(50-66)$ & 0.004 \\
\hline \multicolumn{5}{|l|}{ Radiation field } \\
\hline Involved & $10(13.0)$ & $5(17.2)$ & $5(10.4)$ & 0.489 \\
\hline Extended & $67(87.0)$ & $24(82.8)$ & $43(89.6)$ & \\
\hline \multicolumn{5}{|l|}{ SCN irradiation } \\
\hline Performed & $53(68.8)$ & $22(75.9)$ & $31(64.6)$ & 0.435 \\
\hline Not performed & $24(31.2)$ & $7(24.1)$ & $17(35.4)$ & \\
\hline \multicolumn{5}{|l|}{ Abdominal LN irradiation } \\
\hline Performed & $44(57.1)$ & $9(31.0)$ & $35(72.9)$ & 0.001 \\
\hline Not performed & $33(43.9)$ & $20(69.0)$ & $13(27.1)$ & \\
\hline \multicolumn{5}{|l|}{ ESD } \\
\hline Yes & $26(33.8)$ & $12(41.4)$ & $14(29.2)$ & 0.396 \\
\hline No & $51(66.2)$ & $17(58.6)$ & $34(70.8)$ & \\
\hline \multicolumn{5}{|l|}{ CCRT } \\
\hline Performed & $17(22.0)$ & $9(31.0)$ & $8(16.7)$ & 0.569 \\
\hline Not performed & $60(78.0)$ & $20(69)$ & 40 (83.3) & \\
\hline \multicolumn{5}{|l|}{ Chemotherapy regimen } \\
\hline $5-F U$ & $1(1.3)$ & $1(3.4)$ & 0 & 0.250 \\
\hline 5-FU/cisplatin & $2(2.6)$ & $2(6.9)$ & 0 & \\
\hline Capecitabine & $8(10.4)$ & $4(13.8)$ & $4(8.3)$ & \\
\hline Capecitabine/cisplatin & $3(3.9)$ & $2(6.9)$ & $1(2.1)$ & \\
\hline Paclitaxel/carboplatin & $3(3.9)$ & 0 & $3(6.3)$ & \\
\hline
\end{tabular}

$R T$, radiotherapy; PBT, proton beam therapy; CGE, cobalt gray equivalent; SCN, supraclavicular lymph nodes; LN, lymph nodes; ESD, endoscopic submucosal dissection; CCRT, concurrent chemoradiotherapy; 5-FU, fluorouracil. 

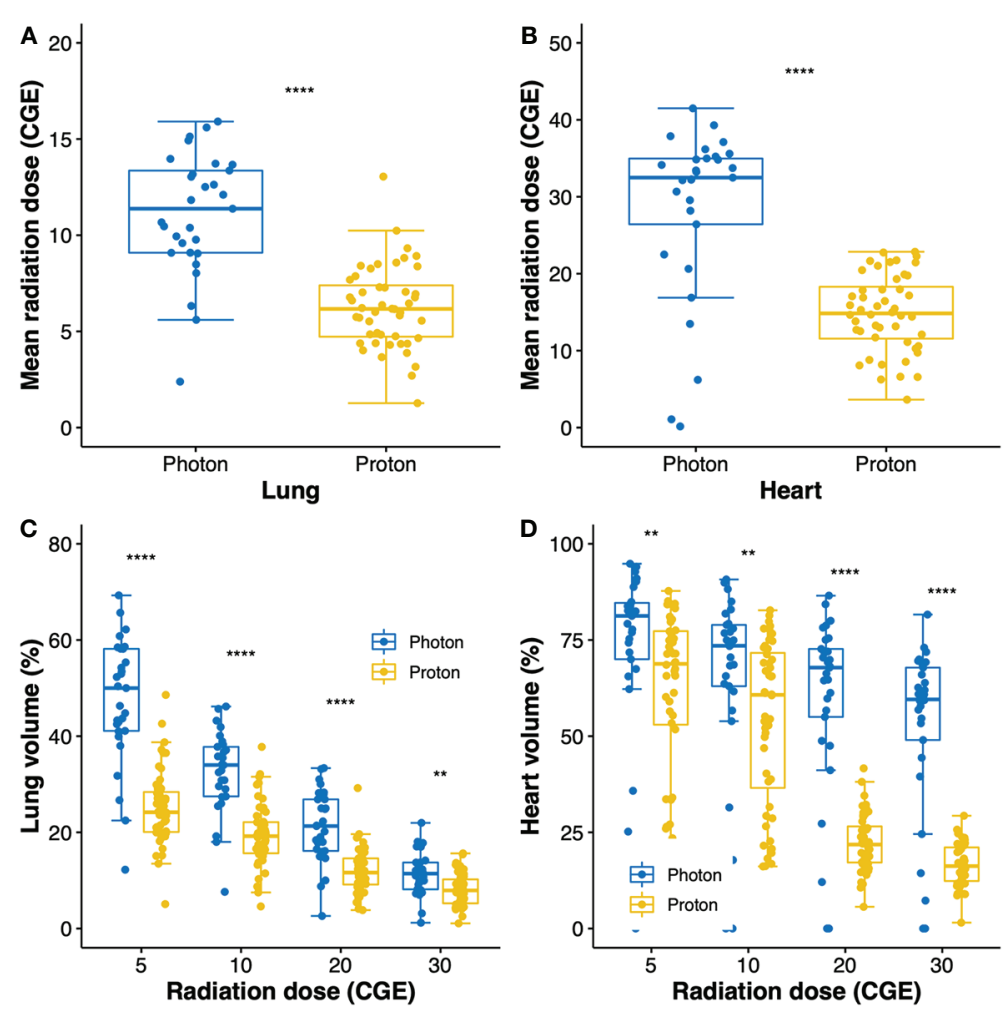

FIGURE 1 | Box plots of mean radiation doses to the lung (A) and heart (B). Box plots showing the distributions of dose-volume indices for the lung (C) and heart (D). Whiskers indicate 1.5 times the interquartile range above and below the mean; dots represent individual observations. CGE, cobalt gray equivalent. ${ }^{* *} p<0.01$, ${ }^{\star \star \star *} p<0.0001$.

reduced the mean radiation doses to the lung (11.1 versus 6.3 CGE, respectively, $p<0.001$; Figure 1A) and heart (32.5 versus 14.8 CGE, respectively, $p<0.001$, Figure 1B). Moreover, the V doses (from V5 to V30) of the lung and heart were significantly lower in the PBT group than in the photon RT group (Figures 1C, D).

\section{Survival and Disease Control}

The median follow-up duration for all patients was 46 (interquartile range, 22-72) months, and those for patients treated with photon RT and PBT were 78 (interquartile range, 69-97) and 25 (interquartile range, 21-42) months, respectively. In all patients, the 5-year progression-free survival (PFS) and overall survival (OS) rates were 56.5 and $64.9 \%$, respectively. There were no significant differences in PFS (Figure 2A) and OS (Figure 2B) between PBT and photon RT. In patients with T1 cancer, the 5-year PFS and OS rates were 62.6\% (Figure 2C) and 73.5\% (Figure 2D), respectively. During the follow-up period, a total of 22 deaths were observed. Among them, 13 were due to disease progression, and one was due to postoperative myocadiac infarction after salvage esophagectomy for local recurrence. The remaining eight were not related to esophageal cancer.

\section{Patterns of Failure Analysis}

Recurrences were observed in 34 sites of 26 patients. All infield recurrences occurred only in the esophagus $(n=19)$; we observed no infield LN metastasis. Among these recurrences, 17 were due to the progression of primary tumors, and two were caused by the development of metachronous primary esophageal cancers. There were three marginal recurrences, of which one and two were detected in the SCN and left gastric LN, respectively. Outfield failures were observed in 12 sites including the upper cervical LN ( $\mathrm{n}=1), \operatorname{SCN}(\mathrm{n}=2)$, mediastinal $\mathrm{LN}(\mathrm{n}=2)$, celiac $\operatorname{LN}(n=2)$, abdominal para-aortic $\operatorname{LN}(n=2)$, liver $(n=2)$, and bone $(n=1)$. In the patient with upper cervical $L N$ recurrence, the SCN was included in the RT field, but regional recurrences occurred at the boundary (SCN) and the outside (upper cervical LN) of the RT field. Two patients, who had received IFRT, experienced outfield mediastinal $\mathrm{LN}$ recurrence. In the two patients who presented with outfield celiac LN recurrence, the celiac LN was not included in the RT field. All solid organ metastases developed synchronously with LN metastasis or consecutively following preceding LN metastasis. The sites of recurrences according to the RT field are shown in Figure 3A. Local, regional, and distant failure rates according to the $\mathrm{T}$ stage are shown in Figures 3B, C. Local failure was most common, followed by regional and distant failures. 
A

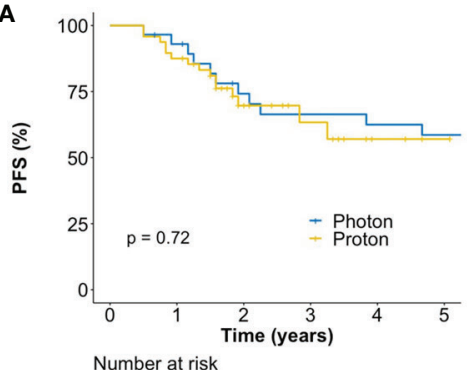

$\begin{array}{lllllll}\text { Photon } 29 & 26 & 19 & 17 & 16 & 15\end{array}$

C

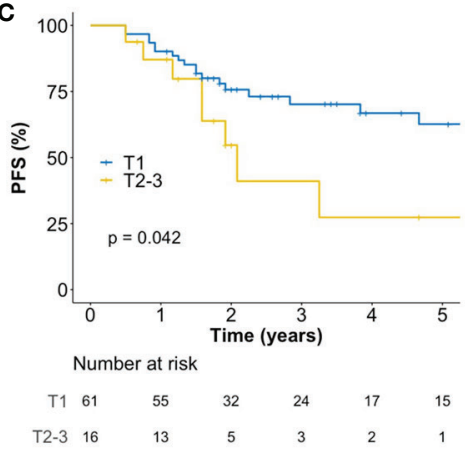

B

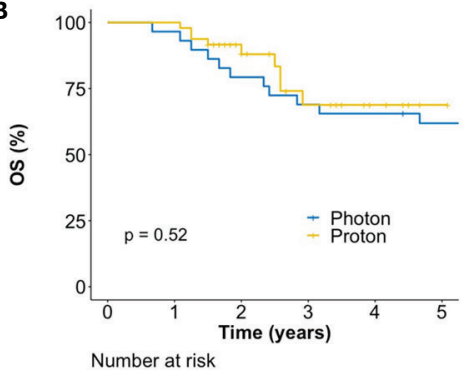

Photon $\begin{array}{llllll}29 & 28 & 23 & 20 & 19 & 17\end{array}$

Proton $\begin{array}{llllll}48 & 48 & 25 & 13 & 5 & 1\end{array}$

D

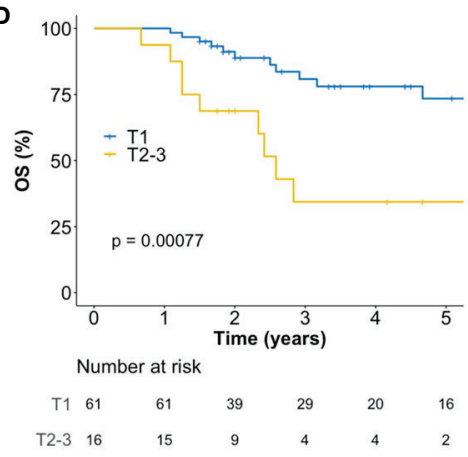

FIGURE 2 | Kaplan-Meier plot of progression-free survival (A) and overall survival (B) rates according to the use of photon RT and PBT in all patients over 60 months. Kaplan-Meier plot of progression-free survival (C) and overall survival (D) rates according to T stage over 60 months. OS, overall survival; PBT, proton beam therapy; PFS, progression-free survival; RT, radiotherapy.

No isolated distant metastasis was without local failure or LN metastasis.

Figure 4 shows the salvage treatments according to failure patterns. Among the 16 patients who experienced isolated local recurrence without regional and distant metastasis, three patients with superficial recurrent cancers were treated endoscopically including argon plasma coagulation $(n=1)$ and ESD $(n=2)$, whereas the 13 patients with deep recurrent cancers underwent esophagectomy $(\mathrm{n}=8)$, chemotherapy $(\mathrm{n}=1)$, and supportive care $(n=4)$. The four patients who presented with regional recurrence without distant metastasis were treated with esophagectomy $(n=1)$, radiotherapy $(n=1)$, chemotherapy $(\mathrm{n}=1)$, and supportive care $(\mathrm{n}=1)$. Of the nine patients who underwent esophagectomy, one patient, who underwent photon RT, died due to postoperative myocadiac infarction, whereas the remaining eight patients (seven with $\mathrm{PBT}$ and one with photon RT) were alive and disease-free until the most recent follow-up. Consequently, among 16 patients with isolated local recurrence, $11(68.8 \%)$ patients were salvaged with esophagectomy or endoscopic treatment. Regional recurrences were only observed at the boundary or outside of the radiotherapy field, never inside this field. However, their salvage rate was poor (20\%).

\section{Treatment-Related Toxicities}

Although almost all patients presented with acute esophagitis, most cases were grade $1(70.1 \%)$. Grade $\geq 2$ acute esophagitis developed significantly more in patients who underwent concurrent chemoradiotherapy $(64.7 \%)$ than in those with RT alone $(20 \%, p=0.001)$. Pleural and pericardial effusion developed in $20(26.0 \%)$ and $11(14.3 \%)$ patients, respectively. Among those, only one photon RT patient, who had a history of paroxysmal atrial fibrillation, required hospitalization owing to pericardial effusion after the completion of concurrent chemoradiotherapy. Among all other patients, no patient presented with grade $\geq 3$ pleural or pericardial effusion. Radiation pneumonitis was observed in 55 patients (71.4\%). However, except for one case with grade 2 radiation pneumonitis, all patients presented with grade 1 radiation pneumonitis. The incident rates of these complications did not differ between the photon RT and PBT groups.

\section{DISCUSSION}

The strategy to reduce RT-related cardiopulmonary toxicity in EC has recently been more emphasized since trimodal therapies like neoadjuvant chemoradiation followed by surgery have been established as a popular standard treatment regimen in most of the operable EC cases $(18,19)$ Therefore, efforts to reduce the radiation dose to organs at risk, especially the lung and the heart, in EC have been made by applying modern RT techniques. As an advanced photon RT technique, IMRT has already shown 
A

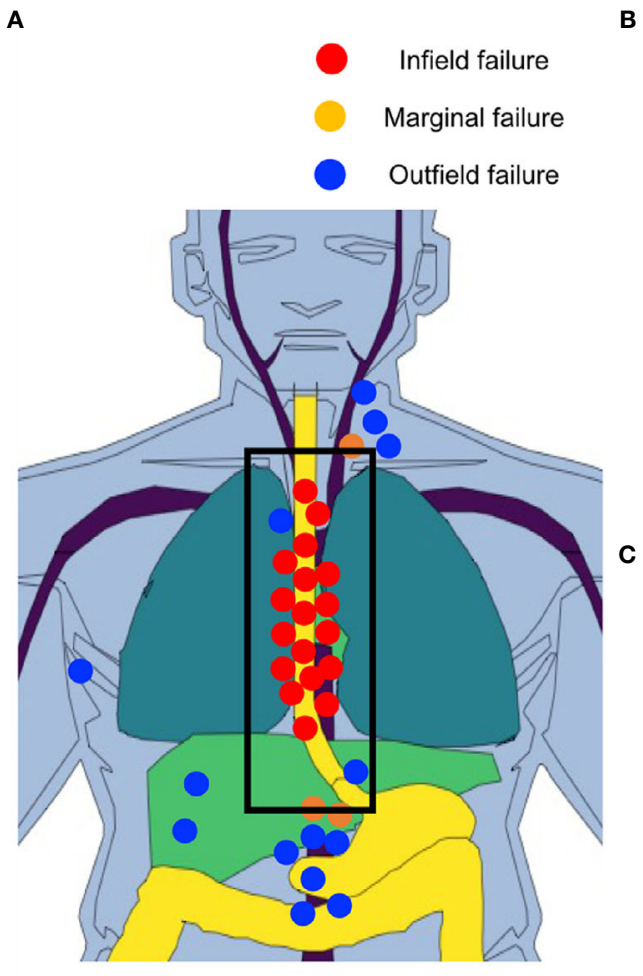

B

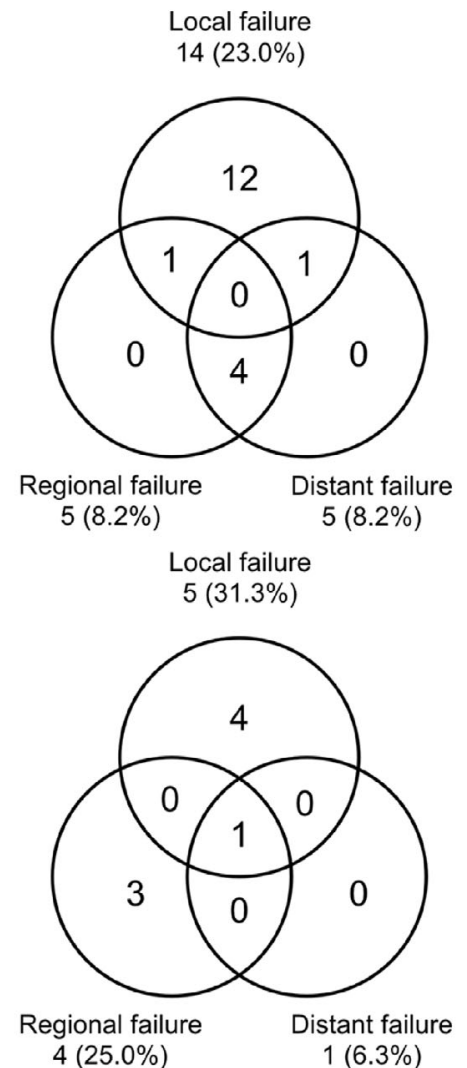

FIGURE 3 | Failure sites according to the RT field in all patients (A). Patterns of treatment failure for patients with T1 (B) and T2-3 (C) cancer. RT, radiotherapy.

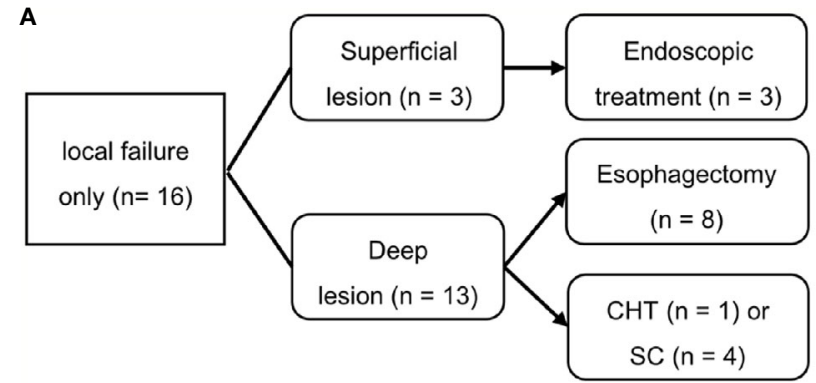

B

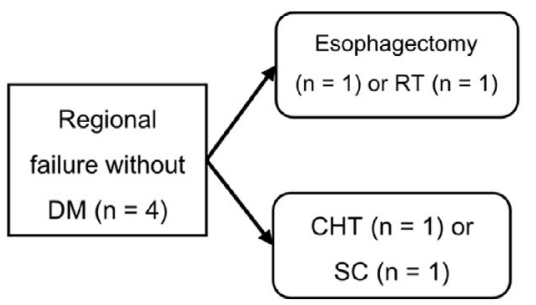

C

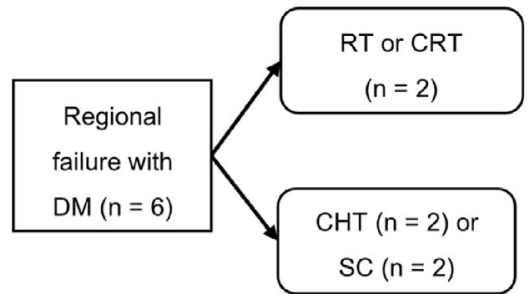

FIGURE 4 | Salvage treatment scheme for patients who experienced isolated local recurrence (A), regional recurrence without distant metastasis (B), or distant metastasis (C). SC, supportive care; CHT, chemotherapy; CRT, chemoradiotherapy; DM, distant metastasis; RT, radiotherapy. 
superior capabilities in comparison to 3D-CRT to decrease radiation exposure to organs at risk in $\mathrm{EC}$ at various locations (20-23), and its application has been associated with significantly higher OS compared to $3 \mathrm{D}$-CRT by reducing cardiac-related deaths (24). PBT has been spotlighted to further reduce the incidence of RT-associated cardiopulmonary complications due to its superior physical properties in comparison to photon RT. Dosimetric studies have already demonstrated that PBT significantly reduces radiation to the whole heart and its substructures, including the left anterior descending artery, left ventricle, and pericardium, compared to 3D-CRT and IMRT (25, 26). In a retrospective study, postoperative pulmonary and GI complication rates were significantly reduced or had the trend towards fewer complications in patients with PBT compared to those with 3D-CRT or IMRT, suggesting that the radiation modality can be an important factor to reduce complication rates in trimodal EC therapy (27).

The present study evaluated treatment outcomes and toxicities of photon RT and PBT for thoracic T1-3 EC without LN metastasis. In the dosimetric comparative analysis, significantly lower radiation doses to organs at risk including the lung and heart were observed in the PBT group compared to the photon RT group. In a previous phase II study comparing IMRT and PBT in definitive or neoadjuvant concurrent chemoradiotherapy for stage II-III esophageal cancer, PBT showed reduced toxicities, but similar PFS compared with IMRT (28). However, in the present study, OS, PFS, and RTrelated toxicities were not significantly different between the photon RT and PBT groups. Although our data did not demonstrate clinical advantages of PBT in terms of clinical outcomes and treatment-related toxicities, the benefits of PBT might be difficult to prove in the current study for the following reasons. The overall toxicity effects in both photon RT and PBT groups seemed to be far from severe, possibly because most of the patients $(78 \%)$ did not receive concurrent chemotherapy. Moreover, a rather long-term follow-up might be needed to evaluate delayed cardiopulmonary toxicity in patients with higher life expectancy, with $79.2 \%$ of all patients being classified as T1. In the previous phase II study, all patients received concurrent chemotherapy, and about half of patients underwent surgery after chemoradiation. These might contribute to higher adverse event rates than our study and evident clinical benefit of PBT in reducing treatment-related toxicities. In the present study, among the patients treated with esophagectomy due to local recurrence, one case of postoperative mortality was observed in the photon RT group, whereas all seven patients treated with PBT successfully underwent salvage esophagectomy without serious postoperative morbidity. As suggested by another study $(27,28)$, PBT might reduce morbidities associated with salvage or planned esophagectomy following neoadjuvant RT, but this is beyond the scope of our analysis.

We also evaluated the effects of the radiation field for T1-3 thoracic EC without LN metastasis in the current study. Notably, EFRT provided good disease control with acceptable toxicity profiles in patients of both photon RT and PBT groups. Although most of the T1 EC patients received EFRT without chemotherapy (90.2\%), their 5-year OS rate was $73.5 \%$ which is comparable to rates previously reported for surgical outcomes (29-32). As there was no clear guideline for RT in T1 EC, we designed our own treatment scheme including the radiation dose, field, and the use of concurrent chemotherapy on the basis of the following hypotheses: 1) a total radiation dose of 6466 Gy without chemotherapy would be sufficient for most of the superficial lesions, similar to the dose for T1 hypopharyngeal cancer; and 2) the elective radiation dose would eradicate clinically invisible LN metastases, similar to the effects observed in hypopharyngeal cancer.

For T1 EC, concurrent chemoradiotherapy is generally considered when esophagectomy is not applicable. Nemoto et al. reported that $\mathrm{RT}$ with or without concurrent chemotherapy resulted in 3-year OS rates of 90 and $70 \%$ for mucosal cancer (T1a) and submucosal cancer (T1b), respectively. OS of patients treated with local RT and concurrent chemotherapy was slightly, although not significantly, better than that of patients treated with ENI without chemotherapy (33). Koide et al. analyzed the treatment outcomes of 60 Gy IFRT combined with concurrent chemotherapy in patients with T1 EC. In this population, IFRT of $60 \mathrm{~Gy}$ with concurrent chemotherapy resulted in 5-year OS, PFS, and local control rates of $77.0,46.9$, and $62.7 \%$, respectively (34). The results of these studies suggest that our outcomes of EFRT without chemotherapy are comparable to those of IFRT with concurrent chemotherapy.

Motoori et al. reported the treatment outcomes of radical esophagectomy and definitive chemoradiotherapy for clinical T1bN0M0 EC (35). They showed that local recurrences after definitive chemoradiotherapy could be controlled with salvage esophagectomy, as observed in all three cases with local recurrence. However, among 20 patients with all types of recurrence, the prognosis of 13 (65\%) patients with LN recurrence was poor after definitive chemoradiotherapy. Only four of these 13 cases could be salvaged with LN dissection or chemoradiotherapy. In our study, 12 patients of the 16 patients with isolated local recurrence could be salvaged with esophagectomy or endoscopic treatment, although one patient treated with photon RT died due to postoperative myocardial infarction. Another case with synchronous local and regional LN recurrence in the left gastric LN was also successfully salvaged by esophagectomy. In our study, regional recurrences were only observed at the boundary or outside of the RT field, never inside the radiation field. Furthermore, all cases of distant LN or solid organ metastasis were detected synchronously with or after the development of regional recurrences (Figures 3B, C). These results indicate that regional control is very important to improve OS in patients with LN-negative EC. This also supports the use of ENI to control subclinical regional diseases within the radiation field.

Although several retrospective studies have demonstrated that concurrent chemoradiotherapy leads to acceptable OS and PFS rates, no prospective study has answered yet the question of whether concurrent chemotherapy is essential, nor has any study compared the efficacy of IFRT and EFRT for T1 EC. Historically, 
concurrent chemotherapy increased the incidence of severe acute toxic effects, as suggested in the RTOG 85-01 study (36). In the current study, EFRT without chemotherapy showed satisfactory treatment outcomes, especially in T1 EC, and acceptable toxicities in the lung and esophagus, whereas the use of chemotherapy tended to increase the risk for grade $\geq 2$ acute esophagitis. This suggests that EFRT without chemotherapy can be considered a treatment option for T1 EC. Esophagectomy is the current standard treatment for patients with lymphovascular or submucosal invasion after ESD. In our study, treatment outcomes were superior in T1 patients who underwent ESD compared to those who did not. These results may be due to a reduced tumor burden after ESD, and/or as an earlier clinical stage without submucosal invasion for patients who underwent ESD. These data indicate that our treatment strategy is applicable to non-curative endoscopic resections.

The survival outcomes of patients with locally advanced EC after definitive chemoradiation are still not satisfactory. Our study confirmed this even in LN-negative EC with mainly local progression, the predominant pattern of failure. Interestingly, this characteristic pattern of lymphatic metastasis was also found in T2-3N0 EC similar to that seen in T1 EC, with a percentage of patients presenting LN metastasis and distant metastasis beyond the RT boundary despite the primary tumor being wellcontrolled. This finding might suggest the necessity of ENI in T2-3N0 EC. Although the importance of regional control in EC was traditionally emphasized in surgical series $(29,32,37)$, the necessity of ENI has also been discussed in definitive RT for T1 EC, as well as in more advanced EC $(12,13)$. A Japanese study group reported promising results of definitive chemoradiation with ENI for stage II-III EC (12). However, hematological or other ENI-associated toxicities might impede the adoption of ENI. In this respect, PBT can be a useful tool to minimize treatment-related toxicities while maintaining the advantage of ENI in the definitive or neoadjuvant CRT setting. PBT may provide even additional benefits related to the reduction in hemato-immunological toxicity. In the propensity scorematched analysis between neoadjuvant PBT and IMRT for EC, patients with PBT developed markedly less frequently grade 4 lymphopenia than those with IMRT, and PBT was found to be an independent predictor of grade 4 lymphopenia, which only trended for poor OS but significantly reduced PFS (16). Other recent studies also demonstrated that lymphopenia is an adverse prognostic factor after chemoradiation in various cancers including EC $(38,39)$ These findings indicate that PBT can improve the survival of EC patients by reducing late treatmentrelated toxicities, as well as radiation-induced lymphopenia.

The limitation of our study are its retrospective and nonrandomized nature and the small number of enrolled patients, because esophagectomy is the standard treatment for T1 EC. In addition, we could not evaluate the patients' quality of life after RT, and the follow-up duration for the PBT group was shorter than that for the photon RT group. Currently, we are conducting a prospective single-arm study at our institution to investigate the efficacy of wider extended-field PBT including almost all lymphatic channels for T1 EC without chemotherapy (Clinical
Research Information Service, Korea Centers for Disease Control and Prevention; KCT0004853).

In conclusion, the dosimetric benefit of significantly reduced radiation doses to the lung and heart in the PBT group compared to the photon RT group did not result in a benefit regarding survival outcome and clinical toxicity in T1-3 EC without LN metastasis. Furthermore, our data demonstrated that EFRT using photon RT and PBT resulted in satisfactory outcomes in patients with T1 EC. Because the characteristics of the lymphatic disease dissemination in T2-3N0 EC were similar to those in superficial EC, we think that EFRT deserves an in-depth investigation regarding locoregional control and overall toxicity in patients with localized EC.

\section{DATA AVAILABILITY STATEMENT}

The raw data supporting the conclusions of this article will be made available by the authors, without undue reservation.

\section{ETHICS STATEMENT}

The studies involving human participants were reviewed and approved by the Institutional Review Board of National Cancer Center in Republic of Korea (NCC2020-0043). Written informed consent for participation was not required for this study in accordance with the national legislation and the institutional requirements.

\section{AUTHOR CONTRIBUTIONS}

Y-GS was responsible for data contribution, data collection, statistical analysis, and manuscript editing. UB was responsible for data collection. $\mathrm{HK}$, JL, MK, and YL were responsible for data contribution. DL was responsible for data collection. SL and TK were responsible for data interpretation. SM was responsible for study design, data contribution, data collection, data interpretation, writing of the manuscript, and all manuscript revisions, and should be considered the guarantor for the article as a whole. All authors contributed to the article and approved the submitted version.

\section{FUNDING}

This study was supported by grants from National Cancer Center Grant (NCC 2110350-1) and the Korea Health Technology R\&D Project through the Korea Health Industry Development Institute (KHIDI) funded by Ministry of Health and Welfare, Republic of Korea (HC19C0293). The funding source had no role in study design, data curation, or analysis and interpretation of the data. 


\section{REFERENCES}

1. Wang Y, Zhu L, Xia W, Wang F. Anatomy of Lymphatic Drainage of the Esophagus and Lymph Node Metastasis of Thoracic Esophageal Cancer. Cancer Manag Res (2018) 10:6295-303. doi: 10.2147/CMAR.S182436

2. Collard JM, Otte JB, Fiasse R, Laterre PF, De Kock M, Longueville J, et al. Skeletonizing En Bloc Esophagectomy for Cancer. Ann Surg (2001) 234 (1):25-32. doi: 10.1097/00000658-200107000-00005

3. Siewert JR, Stein HJ, Feith M, Bruecher BL, Bartels H, Fink U. Histologic Tumor Type Is an Independent Prognostic Parameter in Esophageal Cancer: Lessons From More Than 1,000 Consecutive Resections at a Single Center in the Western World. Ann Surg (2001) 234(3):360-7; discussion 8-9. doi: 10.1097/00000658-200109000-00010

4. Fujita H, Kakegawa T, Yamana H, Shima I, Toh Y, Tomita Y, et al. Mortality and Morbidity Rates, Postoperative Course, Quality of Life, and Prognosis After Extended Radical Lymphadenectomy for Esophageal Cancer. Comparison of Three-Field Lymphadenectomy With Two-Field Lymphadenectomy. Ann Surg (1995) 222(5):654-62. doi: 10.1097/00000658-199511000-00008

5. Nishihira T, Hirayama K, Mori S. A Prospective Randomized Trial of Extended Cervical and Superior Mediastinal Lymphadenectomy for Carcinoma of the Thoracic Esophagus. Am J Surg (1998) 175(1):47-51. doi: 10.1016/S0002-9610(97)00227-4

6. Shim YM, Kim HK, Kim K. Comparison of Survival and Recurrence Pattern Between Two-Field and Three-Field Lymph Node Dissections for Upper Thoracic Esophageal Squamous Cell Carcinoma. J Thorac Oncol (2010) 5 (5):707-12. doi: 10.1097/JTO.0b013e3181d3ccb2

7. Booka E, Takeuchi H, Nishi T, Matsuda S, Kaburagi T, Fukuda K, et al. The Impact of Postoperative Complications on Survivals After Esophagectomy for Esophageal Cancer. Med (Baltimore) (2015) 94(33):e1369. doi: 10.1097/ MD.0000000000001369

8. Nakamura M, Kido Y, Hosoya Y, Yano M, Nagai H, Monden M. Postoperative Gastrointestinal Dysfunction After 2-Field Versus 3-Field Lymph Node Dissection in Patients With Esophageal Cancer. Surg Today (2007) 37(5):379-82. doi: 10.1007/s00595-006-3413-4

9. Jiang L, Zhao X, Meng X, Yu J. Involved Field Irradiation for the Treatment of Esophageal Cancer: Is it Better Than Elective Nodal Irradiation? Cancer Lett (2015) 357(1):69-74. doi: 10.1016/j.canlet.2014.11.045

10. Yamashita H, Takenaka R, Omori M, Imae T, Okuma K, Ohtomo K, et al. Involved-Field Radiotherapy (IFRT) Versus Elective Nodal Irradiation (ENI) in Combination With Concurrent Chemotherapy for 239 Esophageal Cancers: A Single Institutional Retrospective Study. Radiat Oncol (2015) 10:171. doi: 10.1186/s13014-015-0482-9

11. Wang X, Miao C, Chen Z, Li W, Yuan S, Yu J, et al. Can Involved-Field Irradiation Replace Elective Nodal Irradiation in Chemoradiotherapy for Esophageal Cancer? A Systematic Review and Meta-Analysis. Onco Targets Ther (2017) 10:2087-95. doi: 10.2147/OTT.S130285

12. Kato K, Nakajima TE, Ito Y, Katada C, Ishiyama H, Tokunaga SY, et al. Phase II Study of Concurrent Chemoradiotherapy at the Dose of 50.4 Gy With Elective Nodal Irradiation for Stage Ii-III Esophageal Carcinoma. Jpn J Clin Oncol (2013) 43(6):608-15. doi: 10.1093/jjco/hyt048

13. Lee DY, Moon SH, Cho KH, Kim TH, Kim MS, Lee JY, et al. Treatment Outcomes of Extended-Field Radiation Therapy for Thoracic Superficial Esophageal Cancer. Radiat Oncol J (2017) 35(3):241-8. doi: 10.3857/ roj.2017.00458

14. Shiraishi Y, Xu C, Yang J, Komaki R, Lin SH. Dosimetric Comparison to the Heart and Cardiac Substructure in a Large Cohort of Esophageal Cancer Patients Treated With Proton Beam Therapy or Intensity-modulated Radiation Therapy. Radiother Oncol (2017) 125(1):48-54. doi: 10.1016/ j.radonc.2017.07.034

15. Hirano Y, Onozawa M, Hojo H, Motegi A, Zenda S, Hotta K, et al. Dosimetric Comparison Between Proton Beam Therapy and Photon Radiation Therapy for Locally Advanced Esophageal Squamous Cell Carcinoma. Radiat Oncol (2018) 13(1):23. doi: 10.1186/s13014-018-0966-5

16. Shiraishi Y, Fang P, Xu C, Song J, Krishnan S, Koay EJ, et al. Severe Lymphopenia During Neoadjuvant Chemoradiation for Esophageal Cancer: A Propensity Matched Analysis of the Relative Risk of Proton Versus PhotonBased Radiation Therapy. Radiother Oncol (2018) 128(1):154-60. doi: 10.1016/j.radonc.2017.11.028
17. Maag JLV. Gganatogram: An R Package for Modular Visualisation of Anatograms and Tissues Based on Ggplot2. F1000Res (2018) 7:1576. doi: 10.12688/f1000research.16409.1

18. van Hagen P, Hulshof MC, van Lanschot JJ, Steyerberg EW, van Berge Henegouwen MI, Wijnhoven BP, et al. Preoperative Chemoradiotherapy for Esophageal or Junctional Cancer. N Engl J Med (2012) 366(22):2074-84. doi: 10.1056/NEJMoa1112088

19. Nilsson K, Klevebro F, Rouvelas I, Lindblad M, Szabo E, Halldestam I, et al. Surgical Morbidity and Mortality From the Multicenter Randomized Controlled Neores II Trial: Standard Versus Prolonged Time to Surgery After Neoadjuvant Chemoradiotherapy for Esophageal Cancer. Ann Surg (2020) 272(5):684-9. doi: 10.1097/SLA.0000000000004340

20. Chandra A, Guerrero TM, Liu HH, Tucker SL, Liao Z, Wang X, et al. Feasibility of Using Intensity-Modulated Radiotherapy to Improve Lung Sparing in Treatment Planning for Distal Esophageal Cancer. Radiother Oncol (2005) 77(3):247-53. doi: 10.1016/j.radonc.2005.10.017

21. Nutting CM, Bedford JL, Cosgrove VP, Tait DM, Dearnaley DP, Webb S. A Comparison of Conformal and Intensity-Modulated Techniques for Oesophageal Radiotherapy. Radiother Oncol (2001) 61(2):157-63. doi: 10.1016/S0167-8140(01)00438-8

22. Kole TP, Aghayere O, Kwah J, Yorke ED, Goodman KA. Comparison of Heart and Coronary Artery Doses Associated With Intensity-Modulated Radiotherapy Versus Three-Dimensional Conformal Radiotherapy for Distal Esophageal Cancer. Int J Radiat Oncol Biol Phys (2012) 83(5):1580-6. doi: 10.1016/j.ijrobp.2011.10.053

23. Wu VW, Sham JS, Kwong DL. Inverse Planning in Three-Dimensional Conformal and Intensity-Modulated Radiotherapy of Mid-Thoracic Oesophageal Cancer. $\mathrm{Br} J$ Radiol (2004) 77(919):568-72. doi: 10.1259/bjr/19972578

24. Lin SH, Wang L, Myles B, Thall PF, Hofstetter WL, Swisher SG, et al. Propensity Score-Based Comparison of Long-Term Outcomes With 3Dimensional Conformal Radiotherapy vs Intensity-Modulated Radiotherapy for Esophageal Cancer. Int J Radiat Oncol Biol Phys (2012) 84(5):1078-85. doi: 10.1016/j.ijrobp.2012.02.015

25. Zhang X, Zhao KL, Guerrero TM, McGuire SE, Yaremko B, Komaki R, et al. Four-Dimensional Computed Tomography-Based Treatment Planning for Intensity-Modulated Radiation Therapy and Proton Therapy for Distal Esophageal Cancer. Int J Radiat Oncol Biol Phys (2008) 72(1):278-87. doi: 10.1016/j.ijrobp.2008.05.014

26. Taylor CW, Bronnum D, Darby SC, Gagliardi G, Hall P, Jensen MB, et al. Cardiac Dose Estimates From Danish and Swedish Breast Cancer Radiotherapy During 1977-2001. Radiother Oncol (2011) 100(2):176-83. doi: 10.1016/j.radonc.2011.01.020

27. Wang J, Wei C, Tucker SL, Myles B, Palmer M, Hofstetter WL, et al. Predictors of Postoperative Complications After Trimodality Therapy for Esophageal Cancer. Int J Radiat Oncol Biol Phys (2013) 86(5):885-91. doi: 10.1016/j.ijrobp.2013.04.006

28. Dadashova B, Arenas-Ramires B, Mira-McWillaims J, Dixon K, Lord D. Analysis of Crash Injury Severity on Two trans-European Transport Network Corridors in Spain Using Discrete-Choice Models and Random Forests. Traffic Inj Prev (2020) 21(3):228-33. doi: 10.1080/15389588.2020.1733539

29. Tachibana M, Kinugasa S, Yoshimura H, Shibakita M, Tonomoto Y, Dhar DK, et al. Clinical Outcomes of Extended Esophagectomy With Three-Field Lymph Node Dissection for Esophageal Squamous Cell Carcinoma. Am J Surg (2005) 189(1):98-109. doi: 10.1016/j.amjsurg.2004.10.001

30. Pennathur A, Farkas A, Krasinskas AM, Ferson PF, Gooding WE, Gibson MK, et al. Esophagectomy for T1 Esophageal Cancer: Outcomes in 100 Patients and Implications for Endoscopic Therapy. Ann Thorac Surg (2009) 87 (4):1048-54; discussion 54-5. doi: 10.1016/j.athoracsur.2008.12.060

31. Luketich JD, Pennathur A, Awais O, Levy RM, Keeley S, Shende M, et al. Outcomes After Minimally Invasive Esophagectomy: Review of Over 1000 Patients. Ann Surg (2012) 256(1):95-103. doi: 10.1097/SLA.0b013e3182590603

32. Yamamoto S, Ishihara R, Motoori M, Kawaguchi Y, Uedo N, Takeuchi Y, et al. Comparison Between Definitive Chemoradiotherapy and Esophagectomy in Patients With Clinical Stage I Esophageal Squamous Cell Carcinoma. Am J Gastroenterol (2011) 106(6):1048-54. doi: 10.1038/ajg.2011.42

33. Nemoto K, Yamada S, Nishio M, Aoki M, Nakamura R, Matsumoto Y, et al. Results of Radiation Therapy for Superficial Esophageal Cancer Using the Standard Radiotherapy Method Recommended by the Japanese Society of 
Therapeutic Radiology and Oncology (Jastro) Study Group. Anticancer Res (2006) 26(2B):1507-12.

34. Koide Y, Kodaira T, Tachibana H, Tomita N, Makita C, Itoh M, et al. Clinical Outcome of Definitive Radiation Therapy for Superficial Esophageal Cancer. Jpn J Clin Oncol (2017) 47(5):393-400. doi: 10.1093/jjco/hyx021

35. Motoori M, Yano M, Ishihara R, Yamamoto S, Kawaguchi Y, Tanaka K, et al. Comparison Between Radical Esophagectomy and Definitive Chemoradiotherapy in Patients With Clinical T1bN0M0 Esophageal Cancer. Ann Surg Oncol (2012) 19(7):2135-41. doi: 10.1245/s10434-012-2231-8

36. Cooper JS, Guo MD, Herskovic A, Macdonald JS, Martenson JAJr., Al-Sarraf M, et al. Chemoradiotherapy of Locally Advanced Esophageal Cancer: Long-Term Follow-Up of a Prospective Randomized Trial (RTOG 85-01). Radiat Ther Oncol Group JAMA (1999) 281(17):1623-7. doi: 10.1001/jama.281.17.1623

37. Griffin SM, Shaw IH, Dresner SM. Early Complications After Ivor Lewis Subtotal Esophagectomy With Two-Field Lymphadenectomy: Risk Factors and Management. J Am Coll Surg (2002) 194(3):285-97. doi: 10.1016/S10727515(01)01177-2

38. Abravan A, Faivre-Finn C, Kennedy J, McWilliam A, van Herk M. Radiotherapy-Related Lymphopenia Affects Overall Survival in Patients
With Lung Cancer. J Thorac Oncol (2020) 15(10):1624-35. doi: 10.1016/ j.jtho.2020.06.008

39. Davuluri R, Jiang W, Fang P, Xu C, Komaki R, Gomez DR, et al. Lymphocyte Nadir and Esophageal Cancer Survival Outcomes After Chemoradiation Therapy. Int J Radiat Oncol Biol Phys (2017) 99(1):128-35. doi: 10.1016/ j.ijrobp.2017.05.037

Conflict of Interest: The authors declare that the research was conducted in the absence of any commercial or financial relationships that could be construed as a potential conflict of interest.

Copyright (c) 2021 Suh, Bayasgalan, Kim, Lee, Kim, Lee, Lee, Lee, Kim and Moon. This is an open-access article distributed under the terms of the Creative Commons Attribution License (CC BY). The use, distribution or reproduction in other forums is permitted, provided the original author(s) and the copyright owner(s) are credited and that the original publication in this journal is cited, in accordance with accepted academic practice. No use, distribution or reproduction is permitted which does not comply with these terms. 